\title{
Außerunterrichtliche Lern- und Förderarrangements an Ganztagsschulen: Bundesweite Trendanalysen und vertiefende Fallstudien
}

\author{
Johanna May Gaiser ${ }^{1}$, Markus Sauerwein ${ }^{2,3}$, Stephan Kielblock ${ }^{2}$ \\ Justus-Liebig-Universität Gießen \\ 2 DIPF | Leibniz-Institut für Bildungsforschung und Bildungsinformation \\ 3 Fliedner Fachhochschule Düsseldorf
}

\begin{abstract}
Zusammenfassung: Durch erweiterte Zeitfenster haben Ganztagsschulen die Möglichkeit, außerunterrichtliche Lern- und Unterstützungsangebote von Schülerinnen und Schülern anders zu orchestrieren. Durch die Auswertung von repräsentativen Trenddaten einer bundesweiten Schulleitungsbefragung $(N=3509)$ können drei unterschiedliche Klassen der Organisation von Lern- und Förderangeboten an Ganztagsschulen gefunden werden. $\mathrm{Zu}$ diesen drei Klassen wird jeweils eine Fallstudie präsentiert, die ein vertiefendes Verständnis des jeweiligen Lern- und Förderarrangements ermöglicht. Neben Schulen, die sich im Kern auf die Hausaufgabenbetreuung fokussieren (singuläres Arrangement), gibt es auch Schulen, die zusätzlich eine größere Bandbreite an Lernund Förderangeboten bereithalten. Hier erscheint eine inhaltlich-konzeptionelle Vernetzung mit anderen Lern- und Förderangeboten schwierig umzusetzen (paralleles Arrangement). Die dritte Klasse von Schulen sind solche, die ein breites Lern- und Förderangebot mit Lernzeiten kombinieren, wobei sich in der Fallstudie besondere Möglichkeiten einer Vernetzung der unterschiedlichen Lern- und Förderangebote zeigten (vernetztes Arrangement).
\end{abstract}

Schlüsselbegriffe: Ganztagsschule, Lern- und Förderarrangements, (Haus-)Aufgabenangebote, Trendanalysen, Fallstudien

\section{Organization of learning and support activities at German all-day schools: Trend analyses and case studies}

Summary: All-day schools have different opportunities to orchestrate learning and support activities for students by means of extended time slots. Representative data provides three different classes of the organization of learning and support activities in German all-day schools. Each class is described in an exemplary case study to offer a deepened understanding of each arrangement of learning and support activities. Besides schools, which focus only on homework support (singular arrangement), there are also schools offering a wider range of learning and support activities - in addition to homework and homework support. In this class an implementation of a content-related and conceptual interlinking with the other learning and support activities seems to be difficult (parallel arrangement). The third class represents schools, which offer a combination of a wide range of learning and support activities and so-called learning time (individualized learning activity). The associated case study reveals special opportunities for interlinking the different learning and support activities (interlinked arrangement).

Keywords: All-day school, arrangements of learning- and support activities, homework support, trend analysis, case studies

Ganztagsschulen bieten in Deutschland den Schülerinnen und Schülern vielfältige - über den Unterricht hinausgehende - Aktivitäten an
(StEG-Konsortium, 2019). Verstärkt eingeführt wurden Ganztagsschulen in Deutschland unter anderem mit dem Ziel, die individuelle Förde-

Psychologie in Erziehung und Unterricht, 2020, 67, 243-261 
rung zu verbessern und Bildungsungleichheiten zu kompensieren, wobei über den Unterricht hinausgehenden Lern- und Förderangeboten hierfür ein besonderes Potenzial zugeschrieben wird (u. a. BMBF, 2003; Holtappels, 2010; Züchner \& Fischer, 2014). Diese Lern- und Förderangebote stellen einen festen Bestandteil des nicht-curricularen Bereichs von Ganztagsschulen dar. In der bundesweit angelegten Studie zur Entwicklung von Ganztagsschulen (StEG) wurde im Rahmen des seit 2005 durchgeführten Systemmonitorings u. a. die Angebotsstruktur an Ganztagsschulen untersucht. Anhand der Schulleiterbefragung zur Angebotspalette an den befragten Schulen wurde eine Systematisierung von Angebotstypen abgeleitet, welche im Folgenden als theoretischer Ausgangspunkt einer Angebotsgruppierung für diesen Beitrag dient. Die Lern- und Förderangebote lassen sich demnach wie folgt weiter ausdifferenzieren (vgl. dazu u. a. StEG-Konsortium, 2010, S. 22; Furthmüller, 2016; StEGKonsortium, 2019, S. 104ff.): Es kann unterschieden werden zwischen Förderunterricht/ Fördergruppen mit Formen gezielter Lernunterstützung, spezifischen Fördermaßnahmen für bestimmte Schülergruppen, wie z. B. Kinder mit nicht-deutscher Herkunftssprache, fachbezogenen Lernangeboten (mit Bezug zu einem Unterrichtsfach) in Form von Projekten, Werkstätten, AGs/Kursen sowie auch fächerübergreifenden und fachunabhängigen Lernangeboten. Auch wenn eine vollständig trennscharfe Abgrenzbarkeit dieser theoretisch gesetzten Angebotstypen in Bezug auf die Praxis kaum zu erreichen ist, wurden die befragten Schulleitungen für diese Systematisierung sensibilisiert und zuvor eine Liste von Angeboten abgefragt, welche der Logik dieser Systematisierung entspricht (z. B. Naturwissenschaftliche Angebote $=$ fachübergreifende Lernangebote; Deutsch/ Literatur/Lesen $=$ fachbezogene Lernangebote). Eine besondere Art außerunterrichtlicher Lernund Förderangebote stellt der Umgang mit Hausaufgaben bzw. ,neuen' Formen der (Haus-) Aufgaben in der Schule dar. Wenn Hausaufgaben aufgegeben werden, dann gehört fast im- mer auch eine Hausaufgabenbetreuung ins Angebotsrepertoire der Ganztagsschule. Gegenwärtig bieten über 95 Prozent der Ganztagsschulen, die Hausaufgaben aufgeben, auch eine Hausaufgabenbetreuung an (StEG-Konsortium, 2019). Einige Ganztagsschulen haben aufgabenbezogene Lernzeiten (als Selbstlernzeit der Schülerinnen und Schüler) etabliert.

Die jeweils an einem Schulstandort vorfindliche Kombination bestimmter Lern- und Förderangebote wird in diesem Beitrag mit dem Begriff des Lern- und Förderarrangements bezeichnet. Der vorliegende Beitrag nimmt diese außerunterrichtlichen Lern- und Förderarrangements in den Blick, die als ein zentrales Merkmal von Ganztagsschule gelten. Ganztagsschulen können - so eine These dieses Beitrags - basierend auf der Kombination der von ihnen vorgehaltenen Lern- und Förderangebote unterschieden werden. Aus zahlreichen Forschungen ist bereits bekannt, dass es vielfältige außerunterrichtliche Lern- und Förderangebote an Ganztagsschulen gibt (vgl. u.a. StEG-Konsortium, 2019), wie diese in der Praxis an den Schulen konkret arrangiert sind und (inhaltlich) ineinandergreifen, ist bisher ein Forschungsdesiderat. Diesem Themenkomplex widmet sich der vorliegende Beitrag.

\section{Forschungsstand zu Lern- und Förderangeboten an Ganztagsschulen}

Förderunterricht bzw. Fördergruppen und spezifische Fördermaßnahmen für bestimmte Schülergruppen werden an einem Großteil der Ganztagsschulen angeboten. Während an Primarschulen der Anteil der Schulen mit Angeboten im Bereich Förderunterricht/Fördergruppen 2018 bei 76,1 \% lag, war diese Gruppe an Schulen mit Sekundarstufe mit 90,4\% (Sekundarstufe I) bzw. 89,2 \% (Gymnasien) noch häufiger vertreten. Auch spezifische Fördermaßnahmen für bestimmte Schülergruppen sind an Sekundarschulen mit 83,8 \% (Sekundarstufe I) bzw. $76,0 \%$ (Gymnasien) häufiger vertreten als an Primarschulen $(71,9 \%)$. Bei fachbezogenen und fachübergreifenden bzw. fachunabhän- 
gigen Lernangeboten zeigt sich hier ein differenziertes Bild. Im Bereich der fachbezogenen Angebote sind besonders häufig mathematische Angebote, je nach Schulform zwischen 30,3 und $73,3 \%$, und Angebote zu Deutsch/Literatur/Lesen, je nach Schulform zwischen 66,6 und $77,4 \%$, vertreten. Naturwissenschaftliche Angebote bspw. aus der Gruppe der fachübergreifenden Lernangebote sind ebenfalls häufig vorzufinden, nämlich an 51,1 bis $81,7 \%$ der Schulen (StEG-Konsortium, 2019, S. 105ff.). Radisch, Stecher, Klieme und Kühnbach (2008) konnten zeigen, dass die Teilnahme an lernbezogenen Angeboten wie Förderunterricht einen positiven Einfluss auf den individuell wahrgenommenen Lernnutzen von Schülerinnen und Schülern hat. Und umso schülerorientierter die Angebote von den pädagogisch Tätigen gestaltet sind, desto eher schätzten die Schülerinnen und Schüler die Angebote als nützlich für ihre Leistungen und das Lernen ein (S. 256f.). Zumeist, so zeigen aktuelle Untersuchungen, finden sich durch die Teilnahme an den lernbezogenen Angeboten allerdings keine leistungssteigernden Effekte (vgl. hierzu zusammenfassend Sauerwein, Thieme \& Chiapparini, 2019).

Die Hausaufgabenbetreuung gehört an Ganztagsschulen - insbesondere an den Primarschulen $(88,5 \%)$ und Gymnasien (89,7\%) - zu den am häufigsten eingesetzten Lern- und Förderangeboten (StEG-Konsortium, 2019). Eine Hausaufgabenbetreuung findet sich aber grundsätzlich auch an einigen ,Halbtagsschulen', und an Ganztagsschulen wird sie nicht automatisch von allen Schülerinnen und Schülern besucht sie ist also nicht per se ein Spezifikum von Ganztagsschulen.

Viele Schulen sind noch am Anfang ihrer Entwicklung bzgl. der Frage, wie sich Hausaufgaben sinnvoll in die Ganztagsschule integrieren lassen (Grimm \& Schulz-Gade, 2015). Alternativen zur Hausaufgabenbetreuung, wie bspw. in den Vormittag integrierte Lern- und Übungszeiten, haben sich bisher nicht umfassend gegen die traditionelle Form der Hausaufgaben durchgesetzt (Emden, 2016), wie auch die StEG-Schulleitungsbefragung 2018 zeigt. Ein tendenzieller Wechsel in der Organisation der Lernformen kann konstatiert werden: Hausaufgabenbetreuung wird seltener und andere Lern- und Förderangebote etablieren sich (StEG-Konsortium, 2019).

Gestaltungsmöglichkeiten der Lern- und Förderangebote der Schule sind auch an die Organisationsform der Ganztagsschule (gebunden oder offen) geknüpft. So kann an (teil-)gebundenen Ganztagsschulmodellen die (Haus-)Aufgabenbearbeitung in den Vormittag integriert werden (vgl. Höhmann, 2007, 2010). An Schulen, an denen nicht alle Schülerinnen und Schüler am Ganztag teilnehmen, ist es aus organisatorischen Gründen schwieriger, nicht-curriculare Angebote in den Vormittagsbereich zu verlegen, da die Teilnahme an den Angeboten nicht verpflichtend ist (Börner, Gerken, Stötzel \& Tabel, 2013).

Ein Vorteil der Erledigung von (Haus-)Aufgaben in der Schule ist, dass die pädagogisch Tätigen untereinander Lerndefizite ggf. schneller und besser kommunizieren können (Kohler, 2011). Dies erfordert allerdings gute Kommunikation und Kooperation (Höhmann \& Schaper, 2008; Höhmann, 2010). Ist diese nicht gegeben, hat das direkte Konsequenzen für die Umsetzung der Lern- und Fördersettings, sodass die Schülerinnen und Schüler bspw. bei ihren Aufgaben kaum Unterstützung bekommen (Gaiser, Kielblock \& Stecher, 2016).

Bei der Bearbeitung der Hausaufgaben scheint die Quantität der zu erledigenden Aufgaben über der Qualität zu stehen. Die Hausaufgabenbetreuung ist auf eine schnelle und störungsfreie Erledigung der Aufgaben ausgerichtet (ebd.). Auch unterscheiden sich Angebote kaum in ihrer Vermittlungsstruktur vom Unterricht (Stecher, Allemann-Ghionda, Helsper \& Klieme, 2009), sodass die These naheliegt, dass zwischen lernbezogenen Angeboten und dem Unterricht kaum Unterschiede bestehen (Sauerwein, 2017).

Der Fokus auf die Struktur solcher Lernund Förderangebote kann darüber begründet werden, dass sich die Aufgabenerledigung 
(Zepp, 2009) der Schülerinnen und Schüler durch eine strukturierte und effiziente Durchführung verbessert (vgl. dazu auch StEG-Konsortium, 2010). Andererseits handelt es sich beim bloßen Erledigen nur um einen möglichen Modus der (Haus-)Aufgabenorganisation (Gaiser et al., 2016), bei dem ggf. Handlungsfreiheiten für die Schülerinnen und Schüler fehlen (Nordt, 2013) und bei dem kein Beitrag zur Verbesserung der Lernkultur gelingt (Nordt \& Röhner, 2008). Erledigung als maßgebliche Zielgröße erscheint insbesondere deshalb als wenig zielführend, weil bekannt ist, dass Akzep$\operatorname{tanz}$ und Autonomieunterstützung während einer Hausaufgabenbetreuung bzw. aufgabenbezogenen Lernzeit zu einem Lernzuwachs führen (Nordt, 2013). Andere mögliche Modi wären eine Ausrichtung auf Effektivität (mit dem Ziel, nicht nur die Hausaufgaben schnell $\mathrm{zu}$ erledigen, sondern auch gut auf Prüfungen vorbereitet zu sein) oder der Modus der Erweiterung (mit dem Ziel, nicht bloß die Hausaufgaben zu erledigen, sondern die Inhalte wirklich zu verstehen, reflektieren und einzuordnen) (Gaiser et al., 2016). Besonders Letzterer scheint Potenziale zu bieten, die über die einfache Verlängerung bzw. Wiederholung des Unterrichts hinausgehen - vorausgesetzt, das pädagogische Personal bietet kognitiv anregende Aufgaben an.

\section{Herleitung der Forschungsfrage}

Ein Blick auf den Forschungskorpus im Bereich der Lern- und Förderangebote an Ganztagsschulen zeigt, dass Lern- und Förderangebote an Ganztagsschulen weit verbreitet sind. Wenig betrachtet wurde bisher offenbar, wie schulische Strukturen der Lern- und Förderangebote an den jeweiligen Schulen arrangiert sind. Hieraus ergeben sich die Forschungsfragen des vorliegenden Beitrags:

1a) Finden sich interpretierbare Klassen von Ganztagsschulen bzgl. der Organisation der außerunterrichtlichen Lern- und Förderangebote? 1b) Können im zeitlichen Verlauf (2012, 2015 und 2018) unterschiedliche Klassen auf struktureller Ebene bzgl. des Angebots von außerunterrichtlichen Lern- und Förderangeboten beobachtet werden?

Aus dem präsentierten Forschungsstand lässt sich die Hypothese ableiten, dass eine strukturelle Klassifizierung von Lern- und Förderangeboten eng mit der Organisationsform der Ganztagsschulen zusammenhängt. Daher wird zu untersuchen sein:

1c) Hängt die Klassifizierung der Schulen basierend auf Lern- und Förderangeboten mit der Schulform und der Organisationsform des Ganztags zusammen?

Schließlich soll die Analyse von qualitativem Datenmaterial das Bild ergänzen:

2) Wie lassen sich die Lern- und Förderarrangements anhand exemplarischer Fallstudien beschreiben?

\section{Methode}

\section{Forschungsdesign}

Die Forschungsfragen zusammengenommen können nur im Sinne eines Mixed-Methods-Forschungsdesigns (Caracelli \& Greene, 1997) bearbeitet werden. Klassen von Ganztagsschulen mit spezifischem außerunterrichtlichen Lern- und Förderarrangement (vgl. Fragestellung 1a-c) lassen sich hauptsächlich mit quantitativer Methodik herausarbeiten. Hierfür müssten Klassen von Schulen gefunden werden, bei denen innerhalb der jeweiligen Klasse das Vorhalten der Lern- und Förderarrangements auf Schulebene vergleichbar ist, während zwischen den Gruppen benennbare Unterschiede bestehen. Eine andere Herangehensweise erfordert die Analyse der konkreten schulischen Ausgestaltung der Lern- und Förderangebote (vgl. Fragestellung 2). Hier ist eine qualitative Methodik zielführend, die einen schulnahen Blick auf die Praktiken vor Ort erlaubt. Die Verflochtenheit beider Herangehensweisen ist typisch für MixedMethods-Designs (Caracelli \& Greene, 1997), wobei in der vorliegenden Studie die quantitative Analyse die Basis für die Auswahl bestimmter Fälle, die qualitativ vertiefend analysiert werden, bietet. 


\begin{tabular}{|c|c|c|c|c|c|c|c|}
\hline \multicolumn{4}{|c|}{ StEG, 2. Phase (BMBF gefördert) } & \multicolumn{3}{c|}{ StEG, 3. Phase (BMBF gefördert) } \\
\hline 2012 & 2013 & 2014 & 2015 & 2016 & 2017 & 2018 & 2019 \\
\hline $\begin{array}{c}\text { SL- } \\
\text { Befragung } \\
(N=1128)\end{array}$ & $\begin{array}{c}\text { Vertiefungsstudie } \\
\text { an Schulen (StEG-Q) }\end{array}$ & $\begin{array}{c}\text { SL- } \\
\text { Befragung } \\
(N=1236)\end{array}$ & & & $\begin{array}{c}\text { SL- } \\
\text { Befragung } \\
(N=1145)\end{array}$ & \\
\hline
\end{tabular}

Abb. 1: Übersicht zu den Förderphasen und Erhebungszeitpunkten

\section{Daten und Projektkontext}

Hinsichtlich der Forschungsfrage 1 wurden Daten von drei jeweils bundesweit repräsentativen Befragungen von Ganztagsschulleitungen herangezogen (StEGSchulleitungsmonitoring; gefördert vom $\mathrm{BMBF}$ ). Eine detailliertere Darstellung der Stichprobenziehungen findet sich in StEG-Konsortium (2013, 2015, 2019). Diese Befragung wurde 2012, 2015 und 2018 durchgeführt. Die Schulleitungen wurden unter anderem dazu befragt, ob an ihrer Schule Hausaufgaben erteilt werden. Ebenso gaben sie an, ob Hausaufgabenbetreuungen, Lernzeiten, Förderunterricht, spezifische Fördermaßnahmen, fachbezogene Lernangebote sowie fächerübergreifende Lernangebote im Portfolio ihrer Angebote zu finden sind. Insgesamt nahmen 3.509 Schulleitungen an den Befragungen teil.

Bzgl. der Forschungsfrage 2 (Ausgestaltung) wurde auf Daten des Gießener Teilprojekts der Studie zur Entwicklung von Ganztagsschulen (StEG-Q; 2012-2015; gefördert vom BMBF) zurückgegriffen. In StEG-Q wurden mittels Interviews, Gruppendiskussionen und Beobachtungen an neun Ganztagsschulen umfangreiche, längsschnittliche Daten u. a. zu Lern- und Förderangeboten gesammelt. Die Fallauswahl erfolgte basierend auf Ergebnissen der Fragestellung 1 zugrunde liegenden latenten Klassenanalyse. Für die vorliegende Untersuchung wurden Schulen aus StEG-Q ausgewählt, die in ihrer Angebotsstruktur den identifizierten Klassen aus Fragestellung 1 entsprechen. Hierzu wurden systematisierte Informationen zu den Schulen gesichtet, die im Rahmen von StEG-Q erhoben wurden. Anhand einer umfassenden Liste der jeweils angebotenen außerunterrichtlichen Angebote konnten in einem ersten Schritt die Lern- und Förderarrangements pro Schule nachvollzogen werden. Daran anschließend wurden diejenigen Schulen ausgewählt, welche in Bezug auf ihre Angebotspalette den quantitativen Klassen am besten entsprechen. D.h. für Klasse A wurde bspw. eine Schule ausgewählt, bei der die drei Va- riablen mit den höchsten Wahrscheinlichkeiten von annähernd 100\% (Hausaufgaben werden aufgegeben, es werden eine Hausaufgabenbetreuung und Förderunterricht angeboten) als Schulmerkmal gegeben waren, diejenigen mit deutlich niedriger Wahrscheinlichkeit jedoch nicht (vgl. Abbildung 3).

Wie in Abbildung 1 dargestellt, ergibt sich aus den Trendbefragungen der Schulleitungen 2012, 2015 und 2018 in Kombination mit der längsschnittlich angelegten qualitativen Teilstudie StEG-Q, mit drei Feldphasen in 2013 und 2014 ein besonderes Analysepotenzial. Mittels der Schulleitungsdaten kann die Systemveränderung von 2012 zu 2015 nachgezeichnet werden, während die dazwischen liegenden Jahre mit Fallstudien, deren Ausgangsdaten längsschnittlich miteinander verknüpfbar sind, an konkreten Schulstandorten nachgezeichnet werden können. In den Jahren 2016/2017 gibt es keine vergleichbaren qualitativen Daten, sodass der Messzeitpunkt 2018 nicht direkt die qualitativen Daten mit rahmt, sondern der längerfristigen Beschreibung der Trends dient.

\section{Datenerhebung}

\section{Instrumente - StEG-Schulleitungsstudie}

Die Datenerhebung erfolgte zu allen drei Messzeitpunkten unter Nutzung eines Onlinefragebogens. Die Schulleitungen wurden u. a. gebeten anzugeben, ob im aktuellen Schuljahr an ihrer Schule Hausaufgaben aufgegeben werden, ob es eine Hausaufgabenbetreuung und/oder Lernzeiten gibt (ja, nein) und welche unterschiedlichen Angebotsformen es an der Schule gibt (die Ausprägungen nie und selten zusammengefasst sowie häufig und sehr häufig zusammengefasst). In den jeweiligen Items sind Hinweise gegeben, was unter den verschiedenen Kategorien zu verstehen ist (bspw. lautet ein Item „Spezifische Fördermaßnahmen für bestimmte Schülergruppen [z. B. nicht-deutscher Herkunftssprache oder besonderem Förderbedarf]"). 
Tab. 1: Deskriptive Übersicht zur Datenlage bzgl. der Lern- und Förderangebote

\begin{tabular}{|c|c|c|c|c|c|c|c|}
\hline & \multicolumn{2}{|c|}{2012} & \multicolumn{2}{|c|}{2015} & \multicolumn{2}{|c|}{2018} \\
\hline & & $n$ & $\%$ & $n$ & $\%$ & $n$ & $\%$ \\
\hline (HA) & Hausaufgaben erteilt & 1013 & 89,8 & 1073 & 86,8 & 979 & 85,5 \\
\hline (HAB) & Hausaufgabenbetreuung & 981 & 86,9 & 1016 & 82,2 & 889 & 77,7 \\
\hline (ALZ) & Aufgabenbezogene Lernzeit & 595 & 52,7 & 679 & 54,9 & 610 & 53,3 \\
\hline (FöU) & Förderunterricht/-gruppen ${ }^{a}$ & 800 & 70,9 & 851 & 68,9 & 691 & 60,4 \\
\hline (FöM) & Spez. Fördermaßnahmen ${ }^{a}$ & 422 & 37,4 & 550 & 44,5 & 527 & 46,1 \\
\hline (FbL) & Fachbezogene Lernangebote ${ }^{a}$ & 586 & 51,9 & 626 & 50,6 & 613 & 53,5 \\
\hline (FüL) & Fachübergreifende Lernangebote ${ }^{a}$ & 654 & 58,0 & 645 & 52,2 & 693 & 60,5 \\
\hline
\end{tabular}

Anmerkungen: Angaben gewichtet. Nicht getrennt nach Schulformen.

a Die Kategorien ,häufig' und ,sehr häufig' wurden zusammengefasst.

Wie Tabelle 1 zeigt, sind Hausaufgaben und Hausaufgabenbetreuungen an einem Großteil der befragten Ganztagsschulen vorhanden. Auch die anderen abgefragten Lern- und Förderangebote werden an mehr als der Hälfte der Schulen vorgehalten. Lediglich ,spezifische Fördermaßnahmen`im außerunterrichtlichen Bereich sind an weniger als 50 Prozent der befragten Schulen vorhanden.

\section{Feldphasen - Erhebung qualitativer Daten}

Die Datenerhebung im Projekt StEG-Q erfolgte mittels Interviews mit Schülerinnen und Schülern sowie pädagogisch tätigen Erwachsenen, mittels Gruppendiskussionen mit Schülerinnen und Schülern und mittels teilnehmender Beobachtungen. Über einen Zeitraum von zwei Jahren (2013-2014) wurden an insgesamt neun Ganztagsschulen die Datenerhebungen mit einem inhaltlichen Fokus auf die Ganztagsangebote vor Ort durchgeführt. Rekrutiert wurden die Interviewpartnerinnen und -partner mit der Unterstützung der jeweiligen Schulleitung, die einschätzen konnte, welche Personen über die Angebote potenziell Auskunft geben können. Es wurden problemzentrierte Interviews durchgeführt (Kielblock \& Lange, 2013), die davon geleitet waren, was die Interviewten selbst für erzählenswert hielten, die aber gleichsam um Themen kreisten, die für die Studie von besonderer Bedeutung waren (u. a. Beschreibung der außerunterrichtlichen Angebote). Die Interviews dauerten zwischen 10 und 20 Minuten, die Gruppendiskussionen zwischen 10 und 25 Minuten. Für die teilnehmenden Beobachtungen besuchten geschulte Personen die Angebote und fixierten ihre Beobachtungen mithilfe teilstrukturierter Protokolle. Speziell ging es in den offenen Beobachtungen darum, den Ablauf und die sozialen Interaktionen zu beobachten und Notizen dazu anzufertigen (vgl. Lamnek, 2010, S. 500ff). Die Schulen wurden innerhalb des Untersuchungszeitraums drei Mal besucht, wobei bei den wiederholten Erhebungen jeweils inhaltliche Bezüge zu den vorangehenden Interviews hergestellt wurden.

\section{Datenanalyse}

\section{Quantitative Analysen}

Gemäß der ersten Fragestellung interessiert, ob sich interpretierbare Klassen von Ganztagsschulen bzgl. der außerunterrichtlichen Lern- und Förderangebote finden lassen. Innerhalb der jeweiligen Klasse sollten die Lern- und Förderangebote vergleichbar vorzufinden sein, während zwischen den Klassen Unterschiede bestehen (vgl. Fragestellung 1). Latente Klassenanalysen (LCA; aus dem Englischen Latent Class Analysis) bieten ein hierfür geeignetes Verfahren.

In LCAs werden Objekte (hier Ganztagsschulen) anhand ihrer Merkmalsprofile in möglichst wenige Gruppen zusammengefasst, die nach innen hin homogen und nach außen hin möglichst heterogen sind (Giegler \& Rost, 1993). Dabei steht ein Set von Antwortmustern für eine bestimmte Klasse, wobei die Anzahl der zu bestimmenden Klassen zunächst unbekannt ist (Vermunt \& Magidson, 2002; Finch \& Bonk, 2011; Gollwitzer, 2012; Geiser, 2010). Jede Ganztagsschule wird so mit einer bestimmten Wahrscheinlichkeit einer Klasse zugeordnet, wobei in jeder Klasse für die unterschiedlichen Items andere Kategorienwahrscheinlichkeiten gelten (Magidson \& Vermunt, 2004; Kaplan \& Keller, 2011).

Für die Bestimmung der Anzahl der Klassen können unterschiedliche Indikatoren herangezogen werden wie AIC, BIC und aBIC (Nylund, Asparouhov 
\& Muthén, 2007). Letztlich müssen die Klassen aber auch interpretierbar sein. D. h. es gilt Lösungen zu vermeiden, bei denen Klassen entstehen, die nur wenige - in unserem Fall - Schulen repräsentieren (Kaplan \& Keller, 2011; Magidson \& Vermunt, 2004). Ein zeitsparendes und praktikables Vorgehen schlagen Morin, Meyer, Creusier und Biétry (2016) vor. Demnach sollen Fit-Indikatoren grafisch abgebildet werden und wenn es zu keiner bedeutsamen Verbesserung der Fit-Werte kommt (es einen „Knick“ gibt), wird die Klassenlösung akzeptiert, ab der es zu keiner Verbesserung des Fits kam. Für Trenddaten bzw. Gruppenvergleiche besteht zudem die Herausforderung, die Messäquivalenz zu beachten, d.h. es muss geprüft werden, ob zwischen den Analysezeitpunkten die gleiche Anzahl an Klassen vorhanden ist und hieran anschließend die Parameter der einzelnen Gruppen über die Messzeitpunkte hinweg gleichgesetzt werden (Morin et al., 2016). Entsprechend wurden zunächst für die drei Messzeitpunkte getrennte LCAs durchgeführt und dann in einem weiteren Schritt Parameter zwischen den Erhebungszeitpunkten über die drei Messzeitpunkte hinweg gleichgesetzt.

\section{Qualitative Analysen}

In der Bandbreite an unterschiedlichen Angeboten und Schulen, die StEG-Quntersucht hat, wurden Entsprechungen zu den Klassen aus dem Ergebnis zu Forschungsfrage 1 gesucht. Hierzu wurden systematisierte Informationen zu den insgesamt neun untersuchten Schulen gesichtet, die im Rahmen von StEG-Q zusammengetragen wurden. Diese Daten geben u.a. Aufschluss über folgende Punkte: die Schulform, die Organisationsform des Ganztags sowie eine Liste der außerunterrichtlichen Angebote. Insbesondere anhand der Liste der Angebote im Ganztag konnten die Lern- und Förderarrangements pro Schule nachvollzogen werden. Daran anschließend wurden diejenigen Schulen ausgewählt, welche den quantitativen Klassen möglichst gut entsprechen (vgl. zur Auswahl der Fälle 2.2). Hierfür wurde die finale Klassenlösung mit drei Klassen als Ausgangspunkt gewählt und Schulen ausgewählt, die den dort angegebenen Wahrscheinlichkeiten jeweils folgen. War bspw. in Klasse C die Wahrscheinlichkeit, eine aufgabenbezogene Lernzeit anzubieten, sehr hoch (über $90 \%$ ), musste die auszuwählende Schule ebenfalls über ein solches Angebot verfügen.

Schulen, die die jeweiligen quantitativ gebildeten Klassen repräsentieren, wurden daraufhin drei ,explanatorischen Einzelfallstudien ' (Yin, 2009) unterzogen. Hierzu wurden aus dem Datenmaterial diejenigen Quellen ausgewählt, in denen über die Hausaufgaben-, Lern- und Förderangebote Auskunft gegeben wird. Dies ist nicht in allen Daten der Fall, da bei der Erhebung nicht explizit auf die Lernangebote fokussiert wurde, sondern allgemein die Qualität der Angebote an den Schulen thematisiert wurde. Für die Fallstudien ergibt sich auf diese Weise die in Tabelle 2 aufgeführte Datenlage. Die Analyse der Daten umfasste mehrere Schritte. Der Analyse liegt ein Kodierschema zugrunde, welches anhand des Datenmaterials und unter Nutzung der qualitativen Inhaltsanalyse nach Mayring (2015) entwickelt wurde und insgesamt sechs Kategorien umfasst. Pro Fallstudie (auf Schulebene) wurde das Kodierschema deduktiv auf alle Interviews und Beobachtungen angewendet, um das Material inhaltlich zu strukturieren. Hierdurch ließen sich Aussagen zu Struktur, Inhalten, Teilnehmenden, Lernformen, Angebotsleitung und individueller Nutzung der außerunterrichtlichen Angebote bzw. der Lern- und Förderarrangements treffen. Bei der Darstellung der Ergebnisse in Kapitel 3.2 werden an geeigneter Stelle ausgewählte Originalzitate angeführt (vgl. Yin, 2009).

Tab. 2: Fallübersicht: Anzahl der einbezogenen qualitativen Daten

\begin{tabular}{|c|c|c|c|}
\hline Fallstudie & 1 & 2 & 3 \\
\hline Entspricht der Klasse (vgl. Abschnitt 3.1) & A & B & $\mathrm{C}$ \\
\hline Schulform & Gymnasium & $\begin{array}{c}\text { Primarzweig } \\
\text { einer KGS }\end{array}$ & IGS \\
\hline Interviews mit Schülerinnen und Schülern ${ }^{1}$ & 7 & 6 & 7 \\
\hline Gruppendiskussionen Schülerinnen und Schüler & 6 & 3 & 3 \\
\hline Interviews mit päd. tätigem Personal ${ }^{2}$ & 4 & 4 & 4 \\
\hline
\end{tabular}

Anmerkungen: KGS = Kooperative Gesamtschule; IGS = Integrierte Gesamtschule

An allen Schulen waren in den Interviews und Gruppendiskussionen sowohl Mädchen als auch Jungen vertreten (Fallstudie 1: 10-12 Jahre alt, Fallstudie 2: 7-9 Jahre alt, Fallstudie 3: 10-15 Jahre alt).

An allen Schulen waren in den Interviews sowohl Lehrkräfte als auch weiteres päd. tätiges Personal vertreten. 


\section{Ergebnisse}

Im Folgenden werden die detaillierten Ergebnisse ausführlich berichtet. $\mathrm{Zu}$ den quantitativ herausgearbeiteten Klassen der Ganztagsschulen (vgl. Abschnitt 3.1) werden Fallstudien präsentiert, um Komplexität und konkrete Ausgestaltung der Lern- und Förderarrangements in der Praxis zu vertiefen (vgl. Abschnitt 3.2).

\section{Klassen von Ganztagsschulen mit spezifischen außerunterrichtlichen Lern-und Förderarrangements}

\section{Unterscheidung spezifischer Klassen von Ganztagsschulen}

Die Auswahl der Klassen (vgl. Fragestellung 1 a) erfolgte anhand der Fit-Werte (BIC, aBIC und AIC). Die Klassenlösung wurde dabei angenommen, bei der es keine deutliche Verbesserung der Fit-Werte gab („Knick“). Hierfür wurden zunächst für alle drei Erhebungen getrennte Analysen durchgeführt. Abbildung 2 zeigt die Ergebnisse der LCA für die Jahrgänge 2012,
2015 und 2018. Für alle drei Jahrgänge gilt, dass es bis zu drei angenommenen Klassen jeweils zu einer Verbesserung der Fit-Werte (AIC, BIC und aBIC) kommt. Im Vergleich zur Drei-Klassen-Lösung verbessern sich die Fit-Werte bei mehr angenommenen Klassen nicht mehr bedeutsam. Zugleich ist eine drei Klassen umfassende Lösung inhaltlich gut interpretierbar, weshalb für alle drei Zeitpunkte von einer drei Klassen umfassenden Lösung ausgegangen wird.

Weiterhin interessiert, inwiefern die Klassen im Trend vergleichbar sind. Deshalb wurde zunächst, ohne Fixierung von Parametern, für alle drei Jahrgänge eine Drei-Klassen-Lösung berechnet $(\mathrm{AIC}=34.605, \mathrm{BIC}=35.043, \mathrm{aBIC}=34.818$, Entropy $=0,86)$. Es bestehen zwischen den jeweiligen Jahrgängen ähnliche Klassen. Die Wahrscheinlichkeit, dass ein bestimmtes Angebot an einer Schule vorgehalten wird, ist für die jeweiligen Klassen vergleichbar. Beispielsweise ist bei Klasse A zu erkennen, dass in allen drei Erhebungszeiträumen die Hausaufgabenbetreuung an den Schulen mit einer nahezu 100-prozentigen Wahrscheinlichkeit vorgehalten wird,

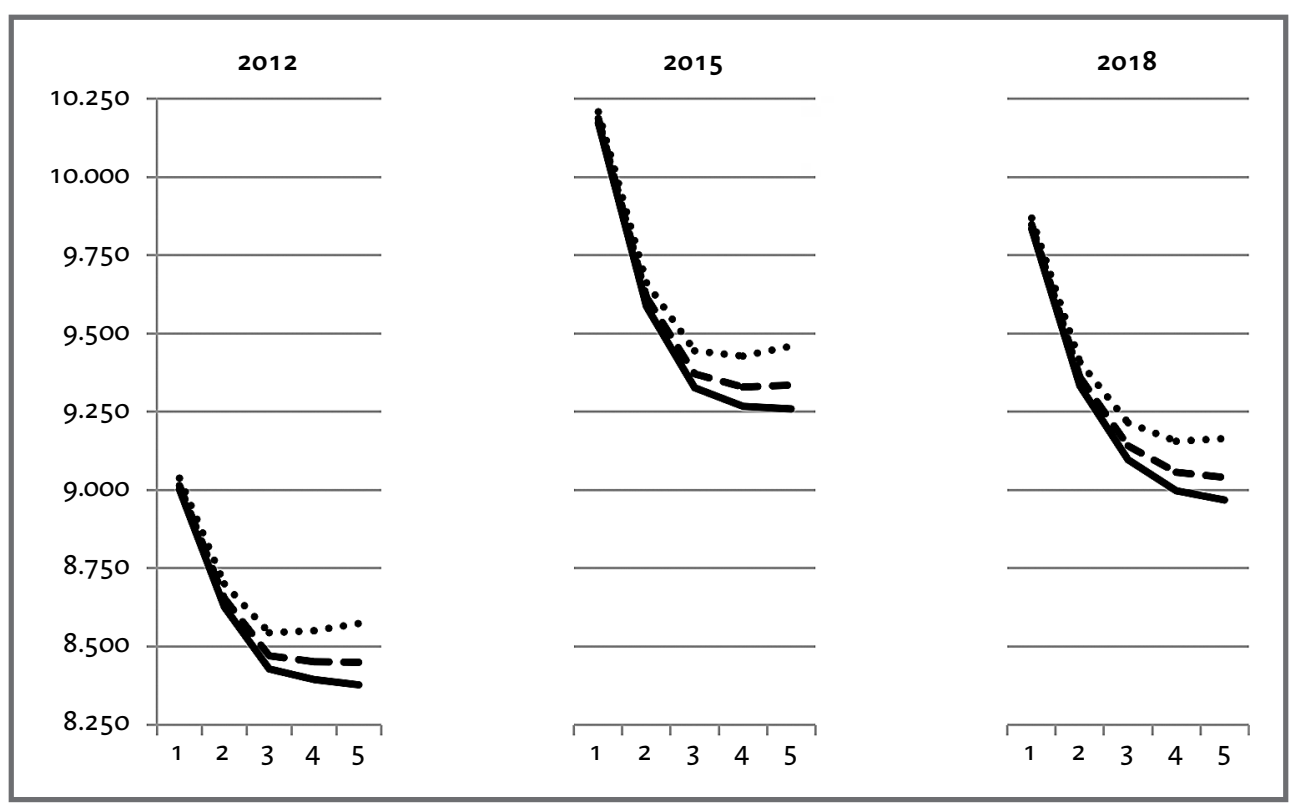

Abb. 2: Fit-Werte der LCA für die drei Messzeitpunkte im Vergleich

Anmerkungen: Fit-Werte der einzelnen Klassenlösungen: $\mathrm{BIC}=$ Punkte; $\mathrm{aBIC}=\mathrm{Striche}$ AIC $=$ Linie 


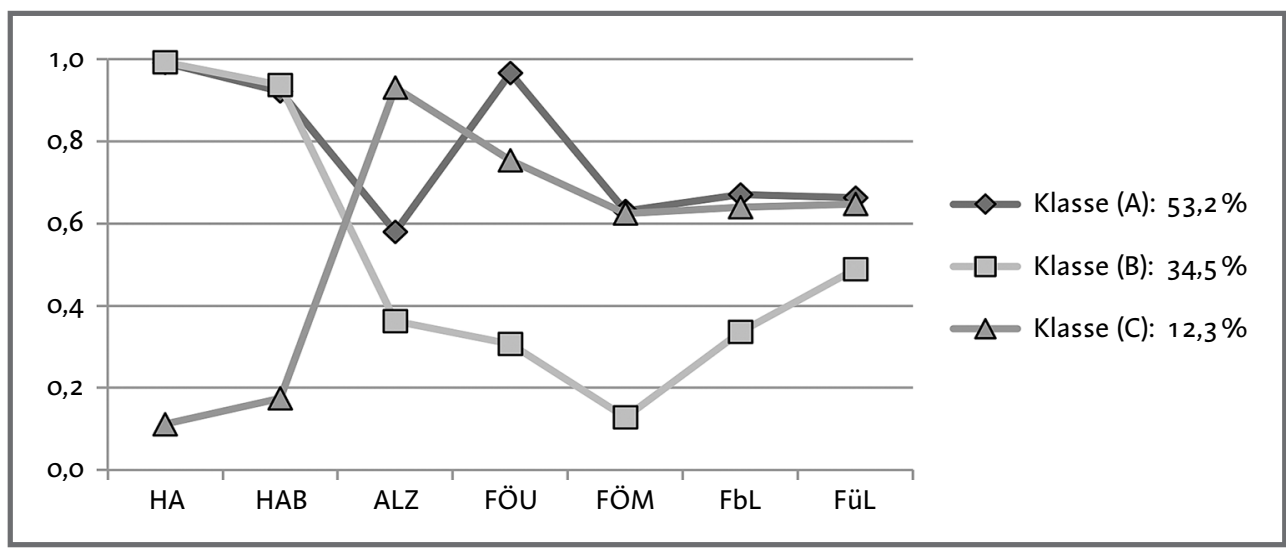

Abb. 3: Finale Klassenlösung

Anmerkungen: $\mathrm{HA}=$ Hausaufgaben erteilt; $\mathrm{HAB}=$ Hausaufgabenbetreuung; $\mathrm{ALZ}=$ Aufgabenbezogene Lernzeit; FöU = Förderunterricht; FöM = Spezifische Fördermaßnahmen; FbL= Fachbezogene Lernangebote; FüL = Fächerübergreifende und fachunabhängige Lernangebote

während Schulen in Klasse B nur bis zur maximal 30-prozentigen Wahrscheinlichkeit auch eine Hausaufgabenbetreuung anbieten.

Um statistisch zu prüfen, inwiefern die Klassen über die drei Zeitpunkte hinweg vergleichbar sind, wurde in einem darauffolgenden Schritt geprüft, ob einzelne Parameter (konkret die Thresholds) jeweils zwischen den Klassen fixiert werden können. Dazu wurden ein Modell mit fixierten Parametern mit dem zuvor beschriebenen unrestringierten Modell verglichen. Das Ergebnis des Vergleichs zeigt, dass für das Modell, in dem die Thresholds zwischen den Gruppen/Jahren gleichgesetzt sind $(\mathrm{AIC}=34.675$, $\mathrm{BIC}=34.854, \mathrm{aBIC}=34.762$, Entropy $=0,85$ ), es zu keiner bedeutsamen Veränderung des Modell-Fits kommt. Entsprechend kann davon ausgegangen werden, dass zwischen den Jahrgängen 2012, 2015 und 2018 drei jeweils vergleichbare Klassen vorliegen. Daraus ergibt sich, dass die Datensätze der drei Messzeitpunkte simultan zur Berechnung der finalen Klassenlösung verwendet werden können.

In Abbildung 3 ist die finale Klassenlösung vorgestellt, bei der die Thresholds über alle drei Messzeitpunkte hinweg fixiert wurden. Anders ausgedrückt: Die drei Klassen wurden aus Daten über alle drei Messzeitpunkte hinweg berechnet. Die erste Klasse (A) umfasst mehr als die Hälfte aller Schulen. Hierbei handelt es sich um Schulen, die Hausaufgaben erteilen und eine Hausaufgabenbetreuung anbieten und auch weitere lernbezogene Angebote. Im Gegensatz dazu zeichnen sich die Schulen der zweiten Klasse (B) dadurch aus, dass nur Hausaufgabenbetreuung angeboten wird, aber nur selten weitere Lernangebote. Im Kontrast zu diesen zwei Schulen stehen die Schulen der dritten Klasse (C) mit aufgabenbezogenen Lernzeiten. Diese umfasst 12,3\% aller Schulen und zeichnet sich dadurch aus, dass keine Hausaufgaben erteilt werden und entsprechend keine Hausaufgabenbetreuung angeboten wird. Dafür werden aufgabenbezogene Lernzeiten, aber auch lernbezogene Angebote bereitgestellt.

\section{Betrachtung der Klassen im Längsschnitt}

Auch wenn es keine Veränderung zwischen den eigentlichen Klassen gibt, zeigt ein differenzierter Blick, dass sich die prozentuale Verteilung der Ganztagsschulen auf die Klassen signifikant verändert hat (vgl. Tabelle 3). So ist ein Rückgang der Schulen mit Hausaufgaben- und Förderangeboten in Relation zu allen anderen Ganztagsschulen festzustellen und ein Anstieg der Schulen mit aufgabenbezogener Lernzeit und der Schulen, die sich auf die Hausaufgabenbetreuung fokussieren. 
Tab. 3: Klassenzugehörigkeit nach Jahren

\begin{tabular}{|l|c|c|c|}
\hline \multicolumn{3}{|c|}{} & \multicolumn{3}{|c|}{ Klassenzugehörigkeit } \\
& (A) & (B) & (C) \\
& HA/B+Förder & HA/B Fokus & ALZ+Lern/Förder \\
\hline 2012 & 57,4 & 32,7 & 9,8 \\
2015 & 55,2 & 32,0 & 12,9 \\
2018 & 47,0 & 38,9 & 14,1 \\
\hline Phi & & $0,095^{* * *}$ & \\
\hline Cramers V & & $0,067^{* * *}$ & \\
\hline
\end{tabular}

Anmerkungen: Zugehörigkeit zu Klasse aus LCA (s. o.); ${ }^{*} p<.05,{ }^{* *} p<.01,{ }^{* * *} p<.001$; Angaben gewichtet in Prozent; $n=3.501$

\section{Betrachtung der Klassen nach Faktoren}

\section{Schulformunterschiede}

Nach Schulformen aufgeteilt, zeigt sich in Tabelle 4, dass Schulen der Sekundarstufe I häufiger ,Lernzeitschulen' sind - und es hier insbesondere zwischen 2012 und 2015 zu einem quantitativen Anstieg kam - dafür aber deutlich seltener reine ,Hausaufgabenschulen' vorhanden sind. Diese Klasse kommt vor allem im Primarbereich vor. Schulen mit Hausaufgaben und Lernangeboten waren bis 2015 in Gymna- sien besonders beliebt, hier ist jedoch ein Rückgang zugunsten der ,Hausaufgabenschulen' zu konstatieren. Dies trifft auch auf Schulen der Sekundarstufe I und Primarschulen zu (wobei Veränderungen an Primarschulen nicht signifikant sind).

\section{Organisationsform}

Nach der Organisationsform des Ganztags betrachtet, fällt auf, dass Lernzeitschulen vor allem Ganztagsschulen mit verpflichtender Teilnahme sind und der Anteil insbesondere zwischen

Tab. 4: Klassenzugehörigkeit nach Jahren und nach Schulform

\begin{tabular}{|c|c|c|c|c|}
\hline & & \multicolumn{3}{|c|}{ Klassenzugehörigkeit } \\
\hline & & $\begin{array}{c}\text { (A) } \\
\text { HA/B + Förder }\end{array}$ & $\begin{array}{c}\text { (B) } \\
\text { HA/B Fokus }\end{array}$ & $\begin{array}{c}\text { (C) } \\
A L Z+\text { Lern/Förder }\end{array}$ \\
\hline \multirow{2}{*}{$\begin{array}{l}\text { PRM } \\
(n=1.271)\end{array}$} & $\begin{array}{l}2012 \\
2015 \\
2018\end{array}$ & $\begin{array}{l}50,2 \\
48,0 \\
43,2\end{array}$ & $\begin{array}{l}44,0 \\
44,3 \\
49,8\end{array}$ & $\begin{array}{l}5,8 \\
7,7 \\
7,0\end{array}$ \\
\hline & $\begin{array}{l}\text { Phi } \\
\text { Cramers V }\end{array}$ & \multicolumn{3}{|c|}{$\begin{array}{l}0,066 \\
0,046\end{array}$} \\
\hline \multirow{2}{*}{$\begin{array}{l}\text { SEK } \\
(n=1.537)\end{array}$} & $\begin{array}{l}2012 \\
2015 \\
2018\end{array}$ & $\begin{array}{l}60,2 \\
57,1 \\
48,9\end{array}$ & $\begin{array}{l}24,7 \\
22,8 \\
28,7\end{array}$ & $\begin{array}{l}15,1 \\
20,1 \\
22,4\end{array}$ \\
\hline & $\begin{array}{l}\text { Phi } \\
\text { Cramers V }\end{array}$ & \multicolumn{3}{|c|}{$\begin{array}{l}0,105^{* * *} \\
0,098^{* * *}\end{array}$} \\
\hline \multirow{2}{*}{$\begin{array}{l}\text { GYM } \\
(n=702)\end{array}$} & $\begin{array}{l}2012 \\
2015 \\
2018\end{array}$ & $\begin{array}{l}64,9 \\
62,7 \\
49,8\end{array}$ & $\begin{array}{l}29,9 \\
31,2 \\
40,3\end{array}$ & $\begin{array}{r}5,2 \\
6,2 \\
10,0\end{array}$ \\
\hline & $\begin{array}{l}\text { Phi } \\
\text { Cramers V }\end{array}$ & \multicolumn{3}{|c|}{$\begin{array}{l}0,095^{* * *} \\
0,067^{* * *}\end{array}$} \\
\hline
\end{tabular}

Anmerkungen: $\mathrm{PRM}=$ Ganztagsschulen mit Primarstufe; SEK = Nicht-gymnasiale Schulen mit Sekundarstufe I; GYM = Ganztagsgymnasien; ${ }^{*} p<.05,{ }^{* *} p<.01,{ }^{* * *} p<.001$; Angaben gewichtet in Prozent 
Tab. 5: Klassenzugehörigkeit nach Jahren und nach Organisationsform

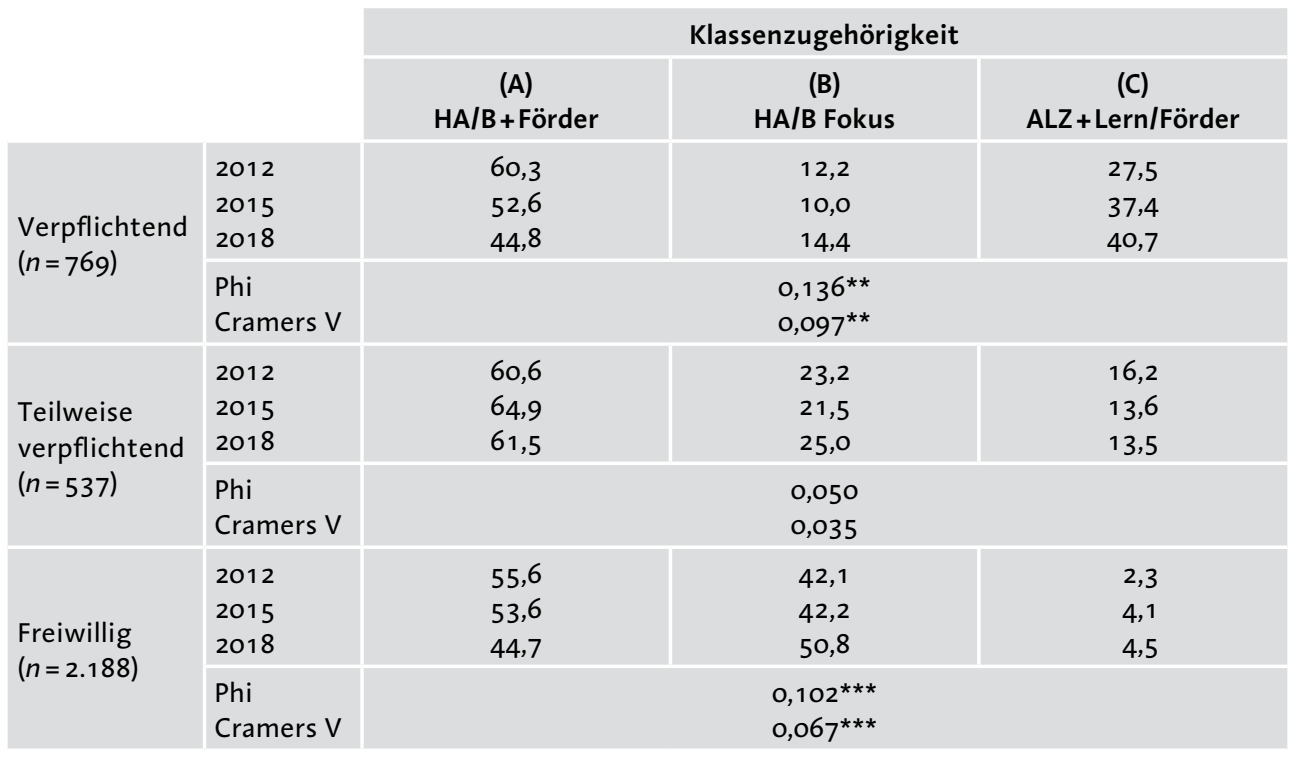

Anmerkungen: PRM = Ganztagsschulen mit Primarstufe; SEK = Nicht-gymnasiale Schulen mit Sekundarstufe I; GYM = Ganztagsgymnasien; Angaben gewichtet in Prozent

2012 und 2015 deutlich gestiegen ist und dafür die Schulen mit Hausaufgaben und Lernangeboten in Relation abgenommen haben (vgl. Tabelle 5). Bei den teilweise Verpflichtenden Schulen sind kaum Veränderungen zu konstatieren. Schulen, an denen die Teilnahme am Ganztag freiwillig ist, sind seltener Lernzeitschulen. Hier überwiegen die ersten beiden Klassen, wobei eine Zunahme an Schulen, die nur eine Hausaufgabenbetreuung anbieten, festzustellen ist.

\section{Qualitative Fallstudien: Ausgestaltung der Lern- und Förderarrangements in der Praxis}

Um die quantitativen Ergebnisse mit den qualitativen Daten zu verknüpfen, wurden drei Schulen ausgewählt, welche die Klassen möglichst gut repräsentieren. In den qualitativen Daten von StEG-Q konnten Schulen gefunden werden, welche mit den jeweiligen Klassen aus der quantitativen Analyse (vgl. 3.1) vergleichbar sind.
Für Klasse A wurde eine Entsprechung mit einem Gymnasium gefunden. An dem ausgewählten Gymnasium werden Hausaufgaben aufgegeben, es wird im teilgebundenen Ganztag eine Hausaufgabenbetreuung angeboten und es gibt für einige Fächer Förderkurse. Dies entspricht der Klasse A in Abbildung 3, bei der mit sehr hoher Wahrscheinlichkeit Hausaufgaben aufgegeben und Hausaufgabenbetreuung sowie Förderunterricht angeboten werden. Klasse B wird von dem Primarzweig einer kooperativen Gesamtschule repräsentiert. Dieser arbeitet im offenen Ganztag und es besteht hierfür eine Kooperation mit einem angegliederten Hort. Es werden Hausaufgaben aufgegeben und die Schülerinnen und Schüler haben die Möglichkeit, diese im Rahmen einer Hausaufgabenbetreuung im Ganztag bzw. im Hort zu erledigen. Von den weiteren lernbezogenen Angebotsvarianten in der quantitativen Analyse ist an diesem Primarzweig keine vorzufinden. Schulen wie diese entsprechen in Abbildung 3 der Linie, die für die Hausaufgaben und die Hausaufgabenbetreuung sehr hohe Werte und für alle anderen 
Angebotsformen sehr geringe Werte aufweist (vgl. Klasse B). Klasse C entspricht in der vorliegenden Untersuchung einer integrierten Gesamtschule, die als teilgebundene Ganztagsschule organisiert ist. An der Gesamtschule werden keine Hausaufgaben aufgegeben und eine aufgabenbezogene Lernzeit findet für die Schülerinnen und Schüler der Jahrgangsstufen 5 bis 7 verpflichtend statt. Förderunterricht in den Hauptfächern wird parallel zu der Lernzeit für bestimmte Fördergruppen angeboten. Als weitere Angebotsform aus der quantitativen Analyse ist an der Schule ein Leseförderangebot (Fachbezogenes Lernangebot Deutsch) vertreten. In Abbildung 3 entspricht diese Schule der Klasse C, die mit hoher Wahrscheinlichkeit eine aufgabenbezogene Lernzeit anbietet und die zudem verschiedene Förderangebote durchführt. Im Folgenden werden diese Fälle zunächst vertiefend analysiert, bevor sie dann in Abschnitt 3.2.4 vergleichend betrachtet werden.

\section{Fallstudie zu Klasse A}

Das zu betrachtende Gymnasium arbeitet als teilgebundene Ganztagsschule. Die Schülerinnen und Schüler bekommen Hausaufgaben auf, die sie entweder zu Hause oder in der Hausaufgabenbetreuung bearbeiten können. Das Angebot ist für alle Schülerinnen und Schüler offen und wird täglich in der 5. bis 9. Unterrichtsstunde angeboten. Die Teilnehmenden können selbst entscheiden, wie viele Stunden sie anwesend sein möchten und nutzen das Angebot dementsprechend flexibel. Das Angebot findet zum einen in der Mediathek statt. Die Angebotsleitung Frau Meyer ${ }^{1}$ unterstützt die Teilnehmenden bei Bedarf bei ihren Aufgaben, ebenso wie einige Oberstufenschülerinnen und -schüler, die zu bestimmten Zeiten anwesend sind.

Neben dem Angebot bei Frau Meyer in der Mediathek gibt es seit einiger Zeit - aufgrund großer Nachfrage - eine Variante des Angebots, in der einzelne Klassen von einer Lehrkraft im Klassenverband bei der Bearbeitung der Aufgaben betreut werden. Auf diese Weise werden komplette Klassen aus dem Angebot in der Mediathek , ausgelagert'. Zu einem regelmäßigen gegenseitigen Austausch über die Schülerinnen und Schüler kommt es zwischen Frau Meyer und den betreuenden Lehrkräften laut Frau Meyer nicht. Sie berichtet, dass sie sich v. a. mit den Schülerinnen und Schülern selbst über die Aufgaben und Lernstände austauscht und „manchmal kommt auch schon was von den Lehrern, oder Kinder werden geschickt [mit dem Hinweis], der muss jetzt mal in die Hausaufgabenbetreuung gehen “". Die beiden Varianten der Hausaufgabenbetreuung existieren weitgehend unverbunden nebeneinander.

Individuelle Förderung und Aktivierung ist in der Hausaufgabenbetreuung ein wichtiges Thema. Brauchen die Teilnehmenden bei den Aufgaben Hilfe, bekommen sie diese von der Angebotsleitung, so erklärt ein Schüler „schön ist auch, dass sie immer nach einem schaut, wenn man Fragen hat, ist nicht alleine, sondern sie ist halt immer da". Es gibt in der Mediathek aber auch Gruppentische, wo sich die Schülerinnen und Schüler gegenseitig helfen können. Aus der Sicht einer Lehrkraft käme in der Hausaufgabenbetreuung in der Mediathek unter den Teilnehmenden ein "Gemeinschaftsgefühl" auf, was sie als großen Vorteil ansieht. Die Angebotsleitung Frau Meyer setzt grundsätzlich voraus, dass die Teilnehmenden ihre Aufgaben selbst organisieren und strukturieren. Sie berichtet, dass im Angebot etwas getan werden müsse: kommt es vor, dass Schülerinnen und Schüler keine Hausaufgaben haben, macht sie diese auf die Lernmaterialien in der Mediathek aufmerksam, mit denen sie weiter üben sollen. Sie macht Vorschläge, was die Teilnehmenden zusätzlich vertiefen oder wiederholen können. Obwohl sie keine Lehrkraft ist, nimmt sie sich selbst als ausreichend fachlich kompetent wahr - und das bestätigen auch die Schülerinnen und Schüler - um diese fachbezogene Lernbegleitung anzubieten.

Pseudonym. Frau Meyer hat als Qualifikation abgeschlossene Studiengänge in Pädagogik/Psychologie und Grafikdesign vorzuweisen. 
Neben der Hausaufgabenbetreuung gibt es an der Schule sowohl Einzel- als auch Gruppenförderunterricht für Kernfächer. Teilweise werden diese Förderkurse von Oberstufenschülerinnen und -schülern (mit) betreut. Eine organisatorische (z. B. zeitlich rhythmisierte) oder inhaltliche Verknüpfung dieser Lern- und Förderangebote mit der Hausaufgabenbetreuung ist an der Schule nicht zu erkennen, weder die Lehrkräfte bzw. weiteren pädagogisch Tätigen noch die teilnehmenden Schülerinnen und Schüler berichten von einer solchen Verknüpfung. Vielmehr werden die Hausaufgabenangebote und der Förderunterricht als zwei verschiedene Alternativen zur Förderung der Schülerinnen und Schüler gesehen, so sagt bspw. die Angebotsleitung der Hausaufgabenbetreuung: „gut, wir haben auch Einzel- und Gruppenförderunterricht, aber man kann das ja mal probieren mit dieser Hausaufgabenbetreuung $[\ldots]$ und da geht auch viel“.

\section{Fallstudie zu Klasse B}

Bei der Fallstudie zu Klasse B handelt es sich um den Primarzweig einer kooperativen Gesamtschule. Der Primarzweig ist als offene Ganztagsschule organisiert. Im Rahmen der sog. Langbetreuung findet das betreute Hausaufgabenangebot für die Schülerinnen und Schüler verpflichtend statt. Die Hausaufgabenbetreuung findet nachmittags in den Räumlichkeiten des angegliederten Horts und unter Aufsicht des Hortpersonals statt. Es stehen zwei Räume zur Verfügung, in denen zwei jahrgangsgemischte Gruppen bei ihren Hausaufgaben betreut werden. Eine Gruppe wurde von dem pädagogischen Personal so eingeteilt, dass dort diejenigen Schülerinnen und Schüler teilnehmen, die durch störendes Verhalten aufgefallen sind. Zudem kommt es gelegentlich - manchmal auch während des laufenden Schuljahrs - zu Personalwechsel in der Hausaufgabenbetreuung.

Eine umfassende individuelle Förderung der Kinder, z. B. in Form einer individuellen Lernberatung zu ergänzenden oder vertiefenden Aufgaben, ist anhand der Daten zur Haus- aufgabenbetreuung nicht zu erkennen. Es geht darum, die Quantität der von den Lehrkräften erteilten Aufgaben zu bewältigen. Hierfür ist das Angebot stark reglementiert, was sich bspw. darin äußert, dass die Schülerinnen und Schüler an Einzeltischen sitzen. Die Teilnehmenden bekommen von den Betreuenden feste Plätze zugewiesen, um zu verhindern, dass sie neben ihren Klassenkameraden sitzen und dass es im Angebot laut wird. Wenn die Schülerinnen und Schüler ihre Aufgaben erledigt haben, melden sie sich bei den Bertreuenden, welche die Aufgaben kontrollieren und einen Eintrag in das Hausaufgabenheft machen. Die Schülerinnen und Schüler müssen bis 15:00 Uhr warten, bevor sie den Raum verlassen dürfen - ganz gleich, ob sie längst fertig sind oder noch Aufgaben zu erledigen haben. Das führt bei den Kindern teilweise zu Langeweile, so sagt ein Schüler: „also wir müssen ja die Hausaufgaben machen [...], aber es ist irgendwie langweilig dann da rumzusitzen, weil da kann man halt nichts so Richtiges machen, wenn man mit den Hausaufgaben fertig ist".

In Einzelfällen sind die Hortangestellten was die Hausaufgabenbetreuung angeht im Austausch miteinander oder ggfs. mit betroffenen Lehrkräften und Eltern: z. B. wenn nachmittags im Hort ein Projekt stattfindet, werden die Lehrkräfte vom Hortpersonal gebeten, wenige oder keine Hausaufgaben aufzugeben, oder eine Lehrkraft weist die Betreuenden darauf hin, dass im Mathematikunterricht das Einmaleins gelernt wird und die Schülerinnen und Schüler bei der Hausaufgabenbetreuung abgefragt werden sollen.

Von zusätzlichen Lern- und Förderangeboten wird in den Interviews an dieser Schule nicht gesprochen. Was dezidierte Lern- und Förderangebote angeht, scheint die Hausaufgabenbetreuung vergleichsweise alleine dazustehen.

\section{Fallstudie zu Klasse C}

Die integrierte Gesamtschule bietet eine teilweise gebundene Form des Ganztags an. Es werden für die Jahrgänge 5 bis 7 keine Hausaufgaben 
aufgegeben. Anstatt dessen findet für diese Jahrgänge vormittags eine zweistündige aufgabenbezogene Lernzeit verpflichtend statt, die von Lehrkräften betreut wird. Neben den Lehrkräften sind auch Schülerinnen und Schüler aus der 9. und 10. Jahrgangsstufe zu bestimmten Zeiten zur Unterstützung bei den Aufgaben vor Ort.

In der Lernzeit wird mit Wochenplan gearbeitet. Begonnen wird jeweils mit einer Stillarbeitsphase von ca. 10 Minuten, in der sich die Teilnehmenden einen Überblick über ihre zu erledigenden Aufgaben verschaffen. Danach dürfen sie Fragen stellen oder im Flüsterton mit anderen an ihren Aufgaben arbeiten. Die Teilnehmenden können entscheiden, ob sie für die Aufgabenbearbeitung im Klassenraum bleiben oder auf den Flur gehen, oder ob sie in den ,Leiseraum' gehen. In einer Beobachtung wurde deutlich, dass während der Lernzeit häufig ein Kommen und Gehen von Kindern ist, die sich bspw. aus ihrem Klassenraum Bücher holen.

Die Teilnehmenden berichten kritisch, dass die jeweiligen Angebotsleitungen die Lernzeit, die ja als möglichst selbstgesteuerte Übungsphase konzipiert ist, teilweise zweckentfremden, indem sie die Schülerinnen und Schüler manchmal dazu anhalten, zuerst die Aufgaben für ihr eigenes Unterrichtsfach zu erledigen.

In Bezug auf die individuelle Förderung unterstützen die Lehrkräfte die Schülerinnen und Schüler bei Bedarf bei ihren Aufgaben. In der Regel ist zur Lernzeit jeweils ein Deutsch-, Englisch- und Mathelehrer vor Ort, der bei Aufgaben zu den jeweiligen Fächern speziell unterstützen kann. Sie setzen sich ggfs. zu einzelnen Kindern, um ihnen bei der Bearbeitung zu helfen, oder bieten ihnen weitere Möglichkeiten der Beschäftigung an, wenn diese ihre Aufgaben bereits erledigt haben.

In der 5. und 6. Jahrgangsstufe wird den Schülerinnen und Schülern in der Lernzeit mehr Struktur gegeben als in den höheren Klassen, da sie die aus Sicht der Lehrkräfte auch brauchen. Das bedeutet, dass in der Lernzeit feste Zeitfenster für die Aufgaben der einzelnen Fächer vorgeschrieben sind. In den höheren Klassenstufen planen die Schülerinnen und Schüler ihre Aufgaben dann zunehmend selbstständig.

In Bezug auf die Kooperation der Lehrkräfte wird aus den Interviews deutlich, dass sie sich untereinander über den aktuellen Stand austauschen, Rücksprachen treffen und die Schülerinnen und Schüler mit speziellen Fragen zu einem Fach ggfs. an einen entsprechenden Fachlehrer weiterverweisen.

Für die Lernzeit wurde eine sog. Forschungsstation in der schuleigenen Mediathek eingerichtet, wo sich Schülerinnen und Schüler, die bereits mit ihren Lernzeitaufgaben fertig sind, weitergehend mit Themen des Unterrichts beschäftigen können. Dort stehen zusätzliche Lernmaterialien zur Verfügung. Zudem gibt es für die Klassen 5 und 6 ein verpflichtendes Leseförderkonzept mit einem Förderkurs, wo die Teilnehmenden Bücher lesen oder eine Lesesoftware nutzen und eine Förderung in Kleingruppen. Außerdem ist von sog. Überhanglehrkräften die Rede, die an zwei Tagen in der Woche zusätzlich bei der Lernzeit unterstützen und die Aufgabe haben, für einzelne Schülerinnen und Schüler mit zusätzlichem Förderbedarf während der Lernzeit in Kleingruppen Förderunterricht zu geben.

\section{Vergleichende Analyse}

Beim systematischen Vergleich der Fallstudien ist besonders augenfällig, dass sich der Grad der Komplexität, wie die Lern- und Förderangebote vor Ort arrangiert sind, stark unterscheidet. Auf der einen Seite kann die Hausaufgabenbetreuung das einzige Lern- und Förderangebot sein, wobei es kaum Querverbindungen zu ähnlichen Angeboten oder dem Unterricht gibt. Auf der anderen Seite kann es ein komplexes Zusammenspiel einer Bandbreite unterschiedlicher Lern- und Förderangebote (auch mit dem Unterricht) geben. Das Ergebnis des Fallvergleichs ist in Tabelle 6 dargestellt und wird im Folgenden beschrieben. 
Tab. 6: Übersicht der Lern- und Förderarrangements

\begin{tabular}{|c|l|c|c|}
\hline Klasse & Quan-Qual-Link' & Lern-/Förderangebot(e) & Arrangement \\
\hline (A) & HA, HAB \& FöU & Unverbunden & Parallel \\
\hline (B) & HA \& HAB & Einzeln & Singulär \\
\hline (C) & ALZ \& weitere & Verschränkt & Vernetzt \\
\hline
\end{tabular}

Quan-Qual-Link meint, welche Charakteristika aus den quantitativen Analysen hinsichtlich der Klassen herausgefunden wurden (quan[titativ]) und dass die qualitative Fallauswahl versucht hat, bewusst diese Charakteristika zu repräsentieren (qual[itativ]).

Bereits anhand von Abbildung 3 lässt sich erahnen, dass die Situation in Klasse B - bezogen auf das Lern- und Förderarrangement - wenig komplex ist. Nahezu alle Schulen dieser Klasse geben Hausaufgaben auf und haben eine Hausaufgabenbetreuung installiert, aber eher wenige dieser Schulen haben weitere Lern- und Förderangebote. In der Fallstudie zu Klasse B zeigt sich, dass es ein ,einzelnes' Lern- oder Förderangebot gibt, welches nicht - oder nur sehr randständig - von anderen vergleichbaren Angeboten flankiert wird. Aufgrund der Fallstudie lässt sich vermuten, dass der vereinzelte Einsatz von Lern- und Förderelementen im außerunterrichtlichen Bereich dann besonders ausgeprägt sein dürfte, wenn das Ganztagskonzept stark additiv umgesetzt wird im Sinne einer Trennung von Vormittag (Schule) und Nachmittag (Hort). Auf das Arrangement der Lern- bzw. Förderangebote bezogen kann in vergleichbaren Fällen also von einem vereinzelten, bzw. singulären Lern- und Förderarrangement' gesprochen werden.

Die Fallstudie zu Klasse A stellt hierzu einen Kontrast dar, da es in Fall A eine gewisse Bandbreite an Lern- und Förderangeboten gibt. Anhand von Abbildung 3 lässt sich belegen, dass einerseits - vergleichbar zu Klasse B - die Hausaufgaben in Kombination mit einer Hausaufgabenbetreuung sehr wahrscheinlich an Schulen aus Klasse A zu finden sind, dass dazu aber häufig (viele) weitere Lern- und Förderangebote angeboten werden. Allerdings wird hier auch deutlich, dass die Hausaufgaben und die Hausaufgabenbetreuung gewissermaßen eine Einheit bilden, die innerhalb des Lernund Förderarrangements kaum ,aufzubrechen ist. Die Hausaufgabenbetreuung hat ihren
Zweck vor allem in der Hausaufgabenerledigung. Dies ist für das Lern- und Förderarrangement bedeutsam, denn die enge Verknüpfung von Hausaufgaben und Hausaufgabenbetreuung ermöglicht kaum eine Öffnung der Hausaufgabenbetreuung gegenüber anderen Lernund Förderangeboten. Dies könnte auch an den Zugzwängen liegen, die die Erledigung der Hausaufgaben mit sich bringt. Nicht zuletzt daher erscheint in den Interviews die Hausaufgabenbetreuung als unabhängig von den vielen weiteren Lern- und Förderangeboten. Da die Lern- und Förderangebote untereinander , unverbunden' sind, kann in Fällen wie dem hier vorgestellten von einem, parallelen Lern- und Förderarrangement' gesprochen werden.

Der substanzielle Unterschied von Klasse C zu den anderen beiden besteht darin, dass keine Hausaufgaben gegeben werden und keine Hausaufgabenbetreuung angeboten wird, sondern dass stattdessen eine Lernzeit installiert worden ist (vgl. Abbildung 3). Die Lernzeit in Fallstudie $\mathrm{C}$ ist eingebunden in und Ausgangspunkt eine/r Bandbreite von außerunterrichtlichen Lern- und Förderangeboten. Diese Angebote sind komplex miteinander verschränkt. Die Lernzeit bringt das Lern- und Förderarrangement näher an den Kernunterricht heran und die Lern- und Fördermaßnahmen, die um die Lernzeit herum und parallel zu ihr laufen, haben ein entsprechendes Gewicht in der Stundentafel; nicht als Zusatz, sondern als ein Herzstück des gesamten LernFörderkonzepts der Schule. Entsprechend kann die Situation in Fallstudie C als ein ,vernetztes Lern- und Förderarrangement' bezeichnet werden. 


\section{Diskussion}

Insgesamt finden sich drei interpretierbare Klassen von Ganztagsschulen bzgl. der Organisation der Lern- und Förderangebote. Im zeitlichen Verlauf (2012, 2015 und 2018) bleibt die Klassenstruktur gleich (vgl. Fragestellung 1b), es ist jedoch eine unterschiedliche Verteilung der jeweiligen Klassen zu beobachten. Die Organisationsform Hausaufgabenbetreuung plus zusätzliche Lerngelegenheiten (Klasse A) ist zwar nach wie vor die quantitativ umfassendste Klasse, die jedoch an Bedeutung verliert, zugunsten von Schulen, die nur auf Hausaufgabenbetreuung setzten (Klasse B) oder von Schulen, die Lernzeiten etablieren (Klasse C). Die eingangs angesprochene Verschiebung der Organisation der Hausaufgaben konnte somit erstmals vertiefend betrachtet werden.

Ferner konnten Zusammenhänge der drei Klassen mit der Organisationsform des Ganztags aufgezeigt werden: Schulen der Sekundarstufe I und Ganztagsschulen mit verpflichtender Teilnahme sind häufiger Schulen, die eine aufgabenbezogene Lernzeit anbieten (Klasse C). Primarschulen sind häufiger als reine ,Hausaufgabenschulen' organisiert, ebenso wie Schulen mit freiwilligen Teilnahmemodellen (Klasse B). Teilweise gebundene Schulen hingegen scheinen häufiger eine Kombination aus Hausaufgaben und Förderangeboten bereitzuhalten (Klasse A).

Diese Klassenspezifika bilden sich in den qualitativen Analysen (Fragestellung 2) dahingehend $\mathrm{ab}$, dass die Ausgestaltungsformen der Lern- und Förderarrangements als, singuläres', ,paralleles und ,vernetztes' Lern- und Förderarrangement bezeichnet werden können. Sie unterscheiden sich insbesondere darin, dass die Lern- und Förderangebote in unterschiedlicher Weise inhaltlich bzw. organisatorisch miteinander verschränkt sind - ein Aspekt, der bei den vorangestellten quantitativen Analysen offengeblieben ist. Das ,singuläre' Lern- und Förderarrangement (Klasse B) kann als additives Angebotskonzept implementiert werden. Für Beaufsichtigung und Kontrolle der Aufgaben in einer vom sonstigen Angebotsrepertoire losgelösten Hausaufgabenbe- treuung wird nur wenig, und nicht zwingend fachlich kompetentes, Personal benötigt. Durch die Abkopplung von Unterricht und Lehrkräften geht es in erster Linie um die schnelle und störungsfreie Erledigung der Aufgaben (vgl. hierzu auch Rabenstein \& Podubrin, 2015).

Das, parallele 'Lern- und Förderarrangement (Klasse A) bietet im Vergleich eine Bandbreite an Angeboten, die in der Organisation ggfs. keinen besonders hohen Aufwand erfordert, da das durchführende Personal nicht notwendigerweise miteinander kooperieren muss. Die Lern- und Förderangebote können in dieser Fallstudie je nach Bedarf genutzt werden, d. h. die Freiwilligkeit bzw. Autonomie seitens der Teilnehmenden ist gut umzusetzen. Durch die unterschiedlichen Durchführenden (Lehrkräfte, weiteres pädagogisches Personal, Oberstufenschülerinnen und -schüler) ist davon auszugehen, dass die Angebote auch unterschiedlich ausgestaltet sind. Der Einsatz unterschiedlicher Personengruppen kann kritisch betrachtet werden, da Haenisch (2009) und Emden (2016) zeigen, dass der Einsatz von Lehrkräften am effektivsten ist. Andererseits weisen Brisson, Sauerwein, Heyl und Theis (2019) gerade im Kontext von Hausaufgabenbetreuung auf die Möglichkeiten des sogenannten Cross-Age Peer Mentoring hin. Ältere Schülerinnen und Schüler haben demnach andere Möglichkeiten, jüngeren unterstützend zur Seite zu stehen.

Im Vergleich der Fallstudien deutet sich an, dass eine komplexere Vernetzung der Lern- und Förderangebote an der Schule mit Hausaufgaben und Hausaufgabenbetreuung nicht so sichtbar war wie an der Schule mit aufgabenbezogener Lernzeit. Dies ist ggf. kein Zufall. Hausaufgaben erzwingen in der Hausaufgabenbetreuung gewisse Praktiken, die die Erledigung der Aufgaben sicherstellen. Dadurch bilden Hausaufgaben und Hausaufgabenbetreuung eine schwer zu durchbrechende Einheit. Dies muss nicht zwingend ein Nachteil sein - aber auf dem Weg zu einem vernetzten Arrangement ist zu überlegen, ob sich ein Umbau der Hausaufgabenbetreuung zu einer Lernzeit nicht lohnen würde. 
In der ,vernetzten 'Variante (Klasse C) sind die verschiedenen Angebote aufeinander abgestimmt und bezogen. Ein mögliches Problem stellt allerdings dar, dass vereinzelt Lehrkräfte die aufgabenbezogene Lernzeit bevorzugt für ihre eigenen Zwecke (für ihr eigenes Fach) nutzen, was auch Rabenstein \& Podubrin (2015) zeigen, und damit auf die eigentlich selbstgesteuerte Aufgabenbearbeitung einwirken. Durch die Verschränkung der unterschiedlichen Angebote, und damit einhergehenden permanenten Kooperationsanforderungen, ist mit einem erhöhten Aufwand seitens der Durchführenden zu rechnen. Dass die Kooperationsbestrebungen im Lern- und Förderarrangement in Fallstudie $\mathrm{C}$ noch auszubauen sind, zeigte sich auch in Interviewaussagen der Angebotsleitungen. Beobachtungen an der in Fallstudie $\mathrm{C}$ beschriebenen Schule gaben zudem Aufschluss darüber, dass bei der Implementation eines so komplexen Angebotsarrangements es auch bei den Teilnehmenden Verwirrung über Zeit, Ort und Fokus der jeweiligen Angebote geben kann. In Rekurs auf die quantitativen Auswertungen zeigt sich, dass diese Variante in ,offenen' Ganztagsschulen kaum anzutreffen ist. Zu begründen ist dies sicherlich über bereits angesprochene organisatorische „Hürden“, die eine Integration von Lernzeiten in den Vormittag erschweren. Teilweise verpflichtende Ganztagsschulen scheinen hier die größte Flexibilität zu gewährleisten, denn hier ist keine Klasse am stärksten repräsentiert.

Dass das singuläre Arrangement an einer Primarschule und das vernetzte Arrangement an einer integrierten Gesamtschule gefunden wurde, ist mit Blick auf die quantitativen Daten $\mathrm{zu}$ erwarten gewesen. Allerdings bedeutet dies nicht, dass die Arrangements an bestimmte Schulformen geknüpft seien. Ebenso wenig sollte in Bezug auf die verschiedenen Lern- und Förderarrangements, wie sie in den Fallstudien beschrieben wurden, Schlüsse über deren grundsätzliche Qualität gezogen werden. Offen bleibt, in welchen Organisationsvarianten das Lernen aus Sicht der Schülerinnen und Schüler am besten gelingt bzw. ein Kompetenzzuwachs am ehesten zu beobachten ist. Hierfür wären weitere Untersuchungen nötig.

Limitierend zeigt sich, dass die hier genutzten Daten vorwiegend Selbstauskünfte der Befragten sind und die tatsächliche Praxis z. B. durch umfangreichere Beobachtungen von Extern nicht untersucht werden konnte. Auch Informationen über die Einbeziehung der Eltern oder weitere, außerschulische Förderangebote wie z. B. Nachhilfe, konnten in diesem Beitrag nicht untersucht werden.

Es konnte auf Systemebene zwar festgestellt werden, dass es einen Rückgang der Schulen mit Hausaufgaben- und Förderangeboten in Relation $\mathrm{zu}$ allen anderen Ganztagsschulen und demgegenüber einen Anstieg der Schulen mit aufgabenbezogener Lernzeit und der Schulen, die sich auf die Hausaufgabenbetreuung fokussieren, gibt. Das bedeutet aber nicht zwingend, dass sich die einzelnen Schulen tatsächlich in die beschriebene Richtung verändern. Die Entwicklungen könnten bspw. auf Systemebene auch durch neu hinzugekommene Ganztagsschulen begründet sein, die zunächst auf die reine Hausaufgabenerledigung setzten. Andererseits spiegeln diese Ergebnisse einen Trend in der Ganztagsschulentwicklung wider. Offene Ganztagsschulen scheinen sich zu etablieren und mögliche pädagogische Potenziale bleiben ungenutzt (StEG-Konsortium, 2019). Allerdings haben auch Ganztagsschulen, die Lernzeiten anbieten, zugenommen, so dass es zumindest einige Schulen gibt, die die zusätzlichen Lernräume des Ganztags nutzen, um das Lernen von Schülerinnen und Schülern anders zu orchestrieren.

Mit der Klassifizierung von Ganztagsschulen bzgl. ihrer Lern- und Förderangebote bietet dieser Beitrag neues Wissen für Forschung und Praxis. Dass die Angebote an den Schulen in verschiedenen ,Arrangements' vorzufinden sind, ist für die Beschreibung von Ganztagsschule auf Systemebene, aber auch für die konkrete Umsetzung in der Praxis hilfreich: die drei beschriebenen Varianten könnten Schulen Ausgangspunkt dafür sein, bewusstere Entscheidungen zu treffen, wenn es um die Aus- und Umgestaltung ihrer Lern- und Förderangebote geht. 


\section{Literatur}

Bundesministerium für Bildung und Forschung (2003). Investitionsprogramm „Zukunft Bildung und Betreuung“. Ganztagsschulen. Zeit für mehr. Bonn.

Börner, N., Gerken, U., Stötzel, J. \& Tabel, A. (2013). Bildungsbericht Ganztagsschule NRW 2013. Institut für soziale Arbeit e.V.

Brisson, B. M., Sauerwein, M. N., Heyl, K. \& Theis, D. (2019). StEG-Tandem: Eine Schulentwicklungsstudie zur Einführung von kooperativen Lernformen in Hausaufgabenbetreuung bzw. Lernzeiten an Ganztagsschulen. Hintergrund, Konzeption und erste Ergebnisse. In S. Maschke, G. Schulz-Gade \& L. Stecher (Hrsg.) Jahrbuch Ganztagsschule 2019/20. Hausaufgaben und Lernzeiten pädagogisch sinnvoll gestalten. Aktuelle Ent wicklungen und Diskussionslinien. Frankfurt a. M.: Debus Pädagogik.

Caracelli, V. J. \& Greene, J. C. (1997). Crafting mixed-method evaluation designs. New Directions for Evaluation 74, 19-32. https://doi.org/10.1002/ev.1069

Emden, M. (2016). Lern- statt Hausaufgaben. Chemielernen im Ganztag. In N. Fischer, H.P. Kuhn \& C. Tillack (Hrsg.), Was sind gute Schulen? Teil 4: Theorie, Praxis und Forschung zur Qualität von Ganztagsschulen (Theorie und Praxis der Schulpädagogik, Band 38 S. 188-200). Immenhausen: Prolog. https://doi.org/ 10.2307/j.ctvddzmj9.15

Finch, W. H. \& Bronk, K. C. (2011). Conducting Confirmatory Latent Class Analysis Using Mplus. Structural Equation Modeling: A Multidisciplinary Journal, 18, 132-151. https://doi.org/10.1080/10705511.2011.5 32732

Furthmüller, P. (2016). Datendokumentation Systemmonitoring 2015: Fragebogen und Datensatz. München.

Gaiser, J. M., Kielblock, S. \& Stecher, L. (2016). Hausaufgabenangebote an Ganztagsschulen. Fallstudien zu Verzahnung von Unterricht und außerunterrichtlichen Angeboten. Zeitschrift für Pädagogik, 62, 797-811.

Geiser, C. (2010). Datenanalyse mit Mplus. Eine anwendungsorientierte Einführung. Wiesbaden: VS. https:// doi.org/10.1007/978-3-531-92042-9

Giegler, H. \& Rost, J. (1993). Typenbildung und Responsesets beim Gießen-Test: Clusteranalyse versus Analyse latenter Klassen. Zeitschrift für Differentielle und Diagnostische Psychologie, 14, 137-152.

Gollwitzer, M. (2012). Latent-Class-Analysis. In H. Moosbrugger \& A. Kelava (Hrsg.), Testtheorie und Fragebogenkonstruktion (S. 295-323). Berlin: Springer. https://doi.org/10.1007/978-3-642-20072-4_12

Grimm, W. \& Schulz-Gade, G. (2015). Übungs-und Lernzeiten an der Ganztagsschule. Ein Praxisleitfaden zur Integration von Hausaufgaben in den Ganztag. Schwalbach: Debus Pädagogik.

Haenisch, H. (2009). Verzahnung zwischen Unterricht und außerunterrichtlichen Angeboten im offenen Ganztag. Eine qualitative Studie zu praktischen Ansätzen der Verzahnung in ausgewählten Schulen. Der GanzTag in NRW - Beiträge zur Qualitätsentwicklung, 5(11).

Höhmann, K. (2007). Organisation von Hausaufgaben in Ganztagsschulen. In K. Höhmann, B. Kohler, Z. Mergenthaler \& C. Wego (Hrsg.), Hausaufgaben an der Ganztagsschule (S. 37-62). Schwalbach: Wochenschau.

Höhmann, K. (2010). Notwendige Last, überflüssiges Ritual oder gewinnbringende Herausforderung? Haus aufgaben an Ganztagsschulen aus Schulleitungssicht.
In H. Buchen, L. Horster \& H.-G. Rolff (Hrsg.) Ganztagsschule: Erfolgsgeschichte und Zukunftsaufgabe (S. 57-70). Stuttgart: Raabe.

Höhmann, K. \& Schaper, S. (2008). Hausaufgaben. In T. Coelen \& H.-U. Otto (Hrsg.), Grundbegriffe Ganztagsbildung. Das Handbuch (S. 576-584). Wiesbaden: VS. https://doi.org/10.1007/978-3-531-91161-8_57

Holtappels, H. (2010). Die Entwicklung von Ganztagsschulen. Konzeptionen, Organisation und pädagogische Gestaltung. In H. Buchen, L. Horster \& H.-G. Rolff (Hrsg.), Ganztagsschule: Erfolgsgeschichte und Zukunftsaufgabe (S. 7-18). Stuttgart: Raabe.

Kaplan, D. \& Keller, B. (2011). A Note on Cluster Effects in Latent Class Analysis. Structural Equation Modeling: A Multidisciplinary Journal, 18, 525-536. https://doi. org/10.1080/10705511.2011.607071

Kielblock, S. \& Lange, A. (2013). Das problemzentrierte Interview: Grundlagen und Forschungspraxis. Zeitschrift für Soziologie der Erziehung und Sozialisation, 33, 439-448.

Kohler, B. (2011). Hausaufgaben. Überblick über didaktische Überlegungen und empirische Untersuchungen. Die Deutsche Schule, 103, 203-218.

Lamnek, S. (2010). Qualitative Sozialforschung (5. Aufl.) Weinheim: Beltz.

Magidson, J. \& Vermunt, J. (2004). Latent Class Models. In D. Kaplan (Ed.), The Sage handbook of quantitative methodology for the social sciences (pp. 175-198). Thousand Oaks: Sage.

Mayring, P. (2015). Qualitative Inhaltsanalyse. Grundlagen und Techniken. Weinheim: Beltz. https://doi.org/10.10 07/978-3-531-18939-0 38

Morin, A. J., Meyer, J. P., Creusier, J. \& Biétry, F. (2016) Multiple-Group Analysis of Similarity in Latent Profile Solutions. Organizational Research Methods, 19, 231 254. https://doi.org/10.1177/1094428115621148

Nordt, G. (2013). Lernen und Fördern in der Hausaufgabenpraxis der offenen Ganztagsgrundschule in NordrheinWestfalen. Eine qualitative Studie aus der Perspektive der pädagogischen Kräfte und der Kinder. Münster: Waxmann.

Nordt, G. \& Röhner, C. (2008). Hausaufgaben in der offenen Ganztagsgrundschule - ein Beitrag zur Förderung des schulischen Lernens und der Schulqualität? Widersprüche, 28(110), 67-79.

Nylund, K. L., Asparouhov, T. \& Muthén, B. O. (2007). Deciding on the Number of Classes in Latent Class Analysis and Growth Mixture Modeling: A Monte Carlo Simulation Study. Structural Equation Modeling: A Multidisciplinary Journal, 14, 535-569. https://doi. org/10.1080/10705510701575396

Rabenstein, K. \& Podubrin, E. (2015). Praktiken individueller Zuwendung in Hausaufgaben und Förderangeboten. Empirische Rekonstruktionen pädagogischer Ordnungen. In S. Reh, B. Fritzsche, T.-S. Idel \& K. Rabenstein (Hrsg.), Lernkulturen. Rekonstruktion pädagogischer Praktiken an Ganztagsschulen (S. 219-263). Wiesbaden: VS. https://doi.org/10.1007/978-3-531 94081-6 14

Radisch, F., Stecher, L., Klieme, E. \& Kühnbach, O. (2018). Unterrichts- und Angebotsqualität aus Schülersicht. In H. G. Holtappels, E. Klieme, T. Rauschenbach \& L. Stecher (Hrsg.), Ganztagsschule in Deutschland. Ergebnisse der Ausgangserhebung der "Studie zur Entwicklung von Ganztagsschulen" (StEG) (S. $227-$ 360). Weinheim: Beltz Juventa. 
Sauerwein, M.N. (2017). Qualität in Bildungssettings der Ganztagsschule. Über Unterrichtsforschung und Sozialpädagogik (1. Auflage). Weinheim: Beltz Juventa.

Sauerwein, M. N., Thieme, N. \& Chiapparini, E. (2019) Wie steht es mit der Ganztagsschule? Ein Forschungsreview mit sozialpädagogischer Kommentierung. Sozia le Passagen, 15, 81-97. https://doi.org/10.1007/s125 92-019-00318-0

Stecher, L., Allemann-Ghionda, C., Helsper, W. \& Klieme, E. (2009) (Hrsg.). Ganztägige Bildung und Betreuung. Zeitschrift für Pädagogik (54. Beiheft). Weinheim: Beltz.

StEG-Konsortium (2010). Ganztagsschule: Entwicklung und Wirkungen. Ergebnisse der Studie zur Entwicklung von Ganztagsschulen 2005-2010. Frankfurt a. M.

StEG-Konsortium (2013). Ganztagsschule 2012/2013. Deskriptive Befunde einer bundesweiten Befragung. Frankfurt a.M.

StEG-Konsortium (2015). Ganztagsschule 2014/2015. Deskriptive Befunde einer bundesweiten Befragung. Frankfurt a.M.

StEG-Konsortium (2019). Ganztagsschule 2017/2018. Deskriptive Befunde einer bundesweiten Befragung. Frankfurt a.M.

Vermunt, J. \& Magidson, J. (2002). Latent class cluster analysis. In J. A. Hagenaars \& A. L. McCutcheon (Eds.) Applied Latent Class Analysis (pp. 89-106). Cambridge: Cambridge University Press. https://doi.org/ 10.1017/CBO9780511499531.004

Yin, R. K. (2009). Case study research. Design and methods. Los Angeles: Sage.

Zepp, L. (2009). Zum Verhältnis von Hausaufgaben und schulischer Leistung(ssteigerung) bei Halbtags- und
Ganztagsschülern. In S. Appel, H. Ludwig, U. Rother \& G. Rutz (Hrsg.), Leben - Lernen - Leisten (S. 103-130). Schwalbach: Wochenschau-Verlag.

Züchner, I. \& Fischer, N. (2014). Kompensatorische Wirkungen von Ganztagsschulen - Ist die Ganztagsschule ein Instrument zur Entkopplung des Zusammenhangs von sozialer Herkunft und Bildungserfolg? Zeitschrift für Erziehungswissenschaft, 17, 349-367. https://doi. org/10.1007/s11618-013-0477-y

\section{Johanna M. Gaiser}

Justus-Liebig-Universität Gießen

Karl-Glöckner-Str. 21 B

35394 Gießen

E-Mail: johanna.m.gaiser@ew.jlug.de

\section{Markus Sauerwein}

Fliedner Fachhochschule Düsseldorf

Geschwister-Aufricht-Str. 9

40489 Düsseldorf

E-Mail: sauerwein@fliedner-fachhochschule.de

\section{Stephan Kielblock}

DIPF | Leibniz-Institut für Bildungsforschung und Bildungsinformation

Rostocker Str. 6

60323 Frankfurt am Main

E-Mail: kielblock@dipf.de 